Amino aci ds $\mathrm{C}$ termi nal to the 14-3 3 bi ndi ng mot if i $n$ CDC25B affect the effi ci ency of $14-3$ bi ndi ng

\begin{tabular}{|l|l|}
\hline 著者 & $\begin{array}{l}\text { Uchi da Sanae, Kubo Aki t sugu, K zu Ryoi chi , } \\
\text { Nakagama Hi t oshi, Nat sunaga Tsukasa, I shi zaka } \\
\text { Yuki hi to, Yanashi ta Kat sumi }\end{array}$ \\
\hline $\begin{array}{l}\text { j our nal or } \\
\text { publ i cat i on t i t l e }\end{array}$ & Journal of Bi ochemi st ry \\
\hline vol une & 139 \\
\hline number & 4 \\
\hline page range & $761-769$ \\
\hline year & $2006-04-01$ \\
\hline URL & ht t p: //hdl . handl e. net $/ 2297 / 14507$ \\
\hline
\end{tabular}




\title{
Amino Acids C-Terminal to the 14-3-3 Binding Motif in CDC25B Affect the Efficiency of 14-3-3 Binding
}

\author{
Sanae Uchida ${ }^{1}$, Akitsugu Kubo ${ }^{1}$, Ryoichi Kizu ${ }^{2, *}$, Hitoshi Nakagama ${ }^{3}$, \\ Tsukasa Matsunaga ${ }^{1}$, Yukihito Ishizaka ${ }^{4}$ and Katsumi Yamashita ${ }^{1,5}$ \\ ${ }^{1}$ Division of Life Science and ${ }^{2}$ Division of Environmental Science and Engineering, Graduate School of Science \\ and Technology, Kanazawa University, Kakuma-machi, Kanazawa, Ishikawa 920-1192; ${ }^{3}$ Biochemistry \\ Division, National Cancer Center Research Institute, 1-1 Tsukiji 5-chome, Chuo-ku, Tokyo 104-0045; \\ ${ }^{4}$ Division of Intractable Disease, International Medical Center of Japan, 21-1 Toyama 1-chome, \\ Shinjyuku-ku, Tokyo 162-8655; and ${ }^{5}$ Cancer Research Institute, Kanazawa University, 13-1 Takara-machi, \\ Kanazawa, Ishikawa 920-0934
}

Received February 8, 2006; accepted February 21, 2006

\begin{abstract}
The phospho-site adapter protein 14-3-3 binds to target proteins at amino acid sequences matching the consensus motif Arg-X-X-Ser/Thr-X-Pro, where the serine or threonine residue is phosphorylated and $X$ is any amino acid. The dual-specificity phosphatase CDC25B, which is involved in cell cycle regulation, contains five 14-3-3 binding motifs, but 14-3-3 preferentially binds to the motif at Ser309 in CDC25B1 (or Ser323 in CDC25B3). In the present study, we demonstrate that amino acid residues $C$-terminal to the 14-3-3 binding motif strongly affect the efficiency of 14-3-3 binding. Alanine substitutions at residues downstream of the Ser309 motif dramatically reduced 14-3-3 binding, although phosphorylation of Ser309 was unaffected. We also observed that binding of endogenous 14-3-3 to mutant CDC25B occurred less efficiently than to the wild type. Mutants to which 14-3-3 cannot bind efficiently tend to be located in the nucleus, although not as specifically as the alanine substitution mutant of Ser309. These results indicate that amino acid sequences $\mathrm{C}$-terminal to the consensus binding site have an important role in the efficient binding of 14-3-3 to at least CDC25B, which may partly explain why some consensus sequences are inactive as 14-3-3 binding sites.
\end{abstract}

Key words: 14-3-3, CDC25B, cell cycle, phosphorylation, subcellular localization.

Abbreviations: CDK, cyclin-dependent kinase; GST, glutathione-S-transferase; MAPKAP, mitogen-activated protein kinase-activated protein; MK2, MAPKAP kinase 2; NLS, nuclear localization signal.

The eukaryotic cell cycle progresses through successive cycles of activation and inactivation of cyclin-dependent kinases (CDKs) that are complexed with cyclins. The activities of these complexes are regulated via several mechanisms, including inhibition of CDK by small proteins (e.g., p16, p21, and p27), inhibitory phosphorylation by Wee1/Myt1 kinases at the ATP binding site of CDK, and activation of dephosphorylation by CDC25 dual-specificity phosphatases.

Three CDC25 phosphatases have been identified in mammalian cells, CDC25A, CDC25B, and CDC25C (1). The first CDC25 phosphatase gene to be identified was that encoding CDC25C, which dephosphorylates phospho-Tyr15 of CDK1 (previously phosphorylated by Wee1 kinase) to promote the $\mathrm{G} 2$ to $\mathrm{M}$ phase transition $(2,3)$. Studies using cultured mammalian cells have suggested that CDC25A plays a role in the $\mathrm{G} 1$ to $\mathrm{S}$ phase transition by activating CDK2/cyclin $\mathrm{E}(4,5)$, and that CDC25B and CDC25C function in M phase entry by activating and maintaining CDK1:cyclin B activity during the M phase (6-9).

*To whom correspondence should be addressed at the present address: Department of Environmental Biochemistry, Faculty of Pharmaceutical Sciences, Doshisha Women's College of Liberal Arts, Kodo, Kyotanabe 610-0395. Tel: +81 76264 5809, Fax: +81 76264 5989, E-mail: katsumi@kenroku.kanazawa-u.ac.jp
Recent reports provided evidence that CDC25A also plays an important role in the G2 to $\mathrm{M}$ phase transition, thus indicating that CDC25A regulates all cell cycle stages (10, 11). However, mice depleted of CDC25C by gene targeting develop normally and become fertile adults (12), and CDC25B knockout mice are born essentially healthy, although the females are sterile because of a defect in oogenesis (13). A recent report confirmed that mice lacking both the $\mathrm{CDC} 25 \mathrm{~B}$ and $\mathrm{CDC} 25 \mathrm{C}$ genes are born healthy and that cells derived from these mice not only undergo normal entry to the $M$ phase but also exhibit no checkpoint defects (14). Therefore, CDC25B and CDC25C are not essential for mouse development and for DNA damage checkpoints.

CDC25 proteins are CDK activators and, as a result, may comprise important cell cycle checkpoints (15-17). When the cell cycle checkpoint kinase CHK1 or CHK2 is activated by genotoxic stress, it phosphorylates several serine or threonine residues in CDC25A, which leads to the ubiquitin-proteasome pathway-mediated degradation of CDC25A that accompanies cell cycle arrest at the G1, G2, or intra-S phase $(10,17)$. CDC25B and CDC25C, as well as CDC25A, are good substrates for CHK1 and CHK2 in vitro (18-20). CDC25B overexpression overrides the G2 checkpoint after ionizing radiation and other genotoxic stresses, and overproduction of a non-phosphorylated 
mutant form of CDC25C partially overrides the G2 checkpoint (21-24).

One of the phospho-site adapter proteins, 14-3-3, appears to be centrally involved in the CDC25 function, especially in terms of checkpoint function (15, 22-25). CHK1 and CHK2 [and very recently mitogen-activated protein kinase-activated protein (MAPKAP) kinase 2 (MK2)] have been shown to phosphorylate CDC25B and CDC25C at phosphorylation sites containing the 14-3-3 consensus binding sequence $(23,24,26)$. The binding of 14-3-3 to CDC25B or CDC25C may recruit these phosphatases to the cytoplasm from the nucleus and help to retain them there, thus preventing CDK1 activation in the nucleus (27-30). However, CDC25 phosphorylation by CHK1 or CHK2 down-regulates its phosphatase activity, in both 14-3-3 binding-dependent and -independent manners (31-33). At this point, the precise role of CDC25 binding to 14-3-3 in the normal cell cycle and its checkpoints remains to be defined.

We previously reported that $14-3-3 \beta$ and $14-3-3 \varepsilon$ can bind to Ser309 phosphorylation site in CDC25B1, and that the single phosphorylation at Ser309 is sufficient to sustain the binding of the $\beta$ and $\varepsilon$ 14-3-3 isoforms (30). In the present study, we further examined the importance of 14-3-3 binding in the regulation of CDC25B. We studied binding to CDC25B of other 14-3-3 isoforms, such as 14-3$3 \gamma, \eta, \theta$, which is also called $\tau$, and $\zeta$ and found that they bind primarily to the Ser309 site. Furthermore, our results reveal major roles not only for the amino acids in the Ser309 consensus site but also for the amino acids surrounding the consensus site in efficient 14-3-3 binding.

\section{EXPERIMENTAL PROCEDURES}

Cell Culture and Transformation-HEK293, Cos-7, and HeLa cells (American Type Culture Collection strains CRL-1573, CRL-1651, and CCL-2, respectively) were grown in Dulbecco's Modified Eagle's Medium supplemented with $10 \%$ fetal bovine serum, $100 \mu \mathrm{g} / \mathrm{ml}$ penicillin, $100 \mathrm{U} / \mathrm{ml}$ streptomycin, and $50 \mu \mathrm{g} / \mathrm{ml}$ M-Plasmocin under a humidified atmosphere of $5 \% \mathrm{CO}_{2}$. Fetal bovine serum, penicillin, and streptomycin were from Sigma (St. Louis, MO, USA), and M-Plasmocin from Invivogen (San Diego, CA, USA). For the transformation of HEK-293 and Cos-7 cells, appropriate amounts of DNA were transfected with FuGENE6 (Roche Diagnostics, Indianapolis, IN, USA). HeLa cells were transfected with LipofectAMINE2000 (Invitrogen, Carlsbad, CA, USA) according to the manufacturer's recommendation.

Plasmid Construction-Plasmids encoding N-terminal double FLAG-tagged CDC25B1, and N-terminal double myc-tagged 14-3-3 $\beta, \varepsilon$, and $\sigma$ were constructed as described previously (30). For the isolation of the other 14-3-3 subtypes, the coding regions were amplified by PCR using specific primers for $14-3-3 \gamma, \eta, \theta$, and $\zeta$. Each amplified fragment was subcloned into a modified pEF6-mycHisB vector (Invitrogen) such that a double Myc-tag was encoded at each $\mathrm{N}$-terminus. The sequences of these primers are shown in Table 1. Human CHK1 and CHK2 were provided by Steve Elledge of Harvard Medical School. Human MK2 cDNA was purchased from Open Biosystems (Huntsville, AL, USA). All were amplified with specific primers (Table 1) and subcloned into modified pEF6mycHisB such that a double HA tag was encoded at the $\mathrm{N}$ - or $\mathrm{C}$-terminus and a $6 \times \mathrm{His}$ tag was encoded at the C-terminus. The GST-CDC25B peptide expression plasmid was constructed by PCR amplification of the CDC25B gene, digestion of the PCR product with NcoI and XhoI, and ligation into the pGEX-KG vector (34). The primers used to amplify CDC25B are shown in Table 1.

Site-Directed Mutagenesis-PCR-based site-directed mutagenesis with complementary oligonucleotide pairs was used to insert alanine point mutations in CDC25B, and to place a BamHI restriction site between Leu319 and Lys320 of CDC25B. The sequence of one strand of each primer pair is shown in Table 2 .

Antibodies-Antisera against a phospho-Ser309 peptide (23) and the FLAG tag (35) were raised as described and affinity purified. Anti-myc and anti-HA antibodies were from Cell Signaling (Beverly, MA, USA), anti-GST

Table 1. PCR primers used for amplification and construction of tagged proteins.

\begin{tabular}{cll}
\hline \multicolumn{1}{c}{ Gene } & Primer & \multicolumn{1}{c}{ DNA sequence $\left(5^{\prime} \rightarrow 3^{\prime}\right)$} \\
\hline $14-3-3 \gamma$ & Forward & AGCCCCGGATCCATGGTGGACCGCGAGCAACTGGTG \\
& Reverse & TCCCCTGAATTCTTAATTGTGCCTTCGCCGCCATC \\
$14-3-3 \eta$ & Forward & CCGAGCCGGATCCCATATGGGGGACCGGGAGCAGCTGCTG \\
& Reverse & CCTGAAGAATTCTCAGTTGCCTTCTCCTGCTTCTTC \\
$14-3-30$ & Forward & CCCGCGGGATCCATGGAGAAGACTGAGCTGATCCAG \\
& Reverse & ACACCCGAATTCGATTTAGTTTCAGCCCCTTCTGC \\
$14-3-3 \zeta$ & Forward & GAACATGGATCCATGGATAAAAAATGAGCTGGTTCAG \\
& Reverse & AAGTTGGAATTCCGGTTAATTTCCCCTCCTTCTTC \\
CHK1 & Forward & CTCGGTCTAGACATGGCAGTGCCCTTTGTG \\
& Reverse & GCCGATGGTGATATCATGTGGCAGGAAGCC \\
CHK2 & Forward & GCTCACGGTACCGCCATGTCTCGGGAGTCG \\
& Reverse & TTCAAACCACGGGATATCCAACACAGCAGC \\
MK2 & Forward & TCCCCGGGTACCATGCTGTCCAACTCCCAGGGCCAG \\
& Reverse & CGCGGTGATATCGTGGGCCAGAGCCGCAGCCTCCAGGG \\
MKK6 & Forward & AAGGGGCATATGTCTCAGTCGAAAGGCAAGAAGCGAAACCCTGGC \\
& Reverse & GTCCACGATATCTTAGTCTCCAAGAATCAGTTTTACAAAAGATGC \\
GST-CDC25B & Forward & GTTCCCCCAGCCATGGAGAGTCTCATTAGT \\
& Reverse & \\
\hline
\end{tabular}


Table 2. Sequences of primer pairs used for PCR-based site-directed mutagenesis of CDC25B.

\begin{tabular}{ll}
\hline \multicolumn{1}{c}{ Mutation } & \multicolumn{1}{c}{ Forward primer sequence $\left(5^{\prime} \rightarrow 3^{\prime}\right)$} \\
\hline Leu304Ala & AAGTGCCAGCGGGCCTTCCGCTCTCCG \\
Arg306Ala & CAGCGGCTCTTCGCCTCTCCGTCCATG \\
Ser309Ala & CTCTTCCGCTCTCCGGCCATGCCCTGCAGC \\
Met310Ala & CGCTCTCCGTCCGCCCCCTGCAGCGTG \\
Pro311Ala & TCTCCGTCCATGGCCTGCAGCGTGATC \\
Cys312Ala & CCGTCCATGCCCGCCAGCGTGATCCGG \\
Ser313Ala & TCCATGCCCTGCGCCGTGATCCGGCCC \\
Val314Ala & ATGCCCTGCAGCGCCATCCGGCCCATC \\
Ile315Ala & CCCTGCAGCGTGGCCCGGCCCATCCTC \\
Arg316Ala & TGCAGCGTGATCGCTCCCATCCTCAAG \\
Pro317Ala & AGCGTGATCCGGGCCATCCTCAAGAGG \\
Ile318Ala & GTGATCCGGCCCGCCCTCAAGAGGCTG \\
Leu319Ala & ATCCGGCCCATCGCCAAGAGGCTGGAG \\
319/BamHI/320 & CGGCCCATCCTCGGATCCAAGAGGCTGGAG \\
\hline
\end{tabular}

serum from Amersham Biosciences (Piscataway, NJ, USA), and anti-FLAG M2-beads from Sigma.

Preparation of Cell Extracts, Immunoprecipitation, and Immunoblotting-Crude cell extracts were prepared as described previously (30). Cells were collected by scraping, washed in ice-cold phosphate-buffered saline (PBS), and lysed with immunoprecipitation (IP) buffer $(50 \mathrm{mM}$ Tris-HCl, pH 7.5, $150 \mathrm{mM} \mathrm{NaCl}, 0.5 \% \mathrm{NP}-40,5 \mathrm{mM}$ EGTA, and $1 \mathrm{mM}$ EDTA) supplemented with protease and phosphatase inhibitor mixes as described previously (30). Cell extracts were centrifuged at $15,000 \times \mathrm{g}$ for $10 \mathrm{~min}$ at $4^{\circ} \mathrm{C}$, the supernatant fractions were collected, and the protein concentrations were determined by the Bradford method (Bio-Rad, Richmond, CA, USA) (36). For immunoprecipitation, typically $500 \mu \mathrm{g}$ of protein was mixed with anti-FLAG M2-agarose beads or $2 \mu \mathrm{g}$ of anti-myc monoclonal antibodies followed by protein G-Sepharose beads (Amersham Biosciences). Crude cell extracts or immunoprecipitates were subjected to immunoblotting using rabbit polyclonal anti-FLAG antibodies (for CDC25B), mouse monoclonal anti-myc antibodies (for 14-3-3), or mouse monoclonal anti-HA antibodies (for kinases).

Protein Purification-Protein kinases were prepared from cDNA-transfected Cos-7 cells. Typically, $8 \times 10^{5}$ Cos-7 cells in a $6-\mathrm{cm}$ dish received $4 \mu \mathrm{g}$ of plasmid DNA with FuGENE6. After $24 \mathrm{~h}$, the cells were collected, washed with PBS, and lysed with EDTA-free IP buffer supplemented with a 1:100 dilution of FOCUS Protease Arrest (EMD Biosciences, San Diego, CA, USA), $20 \mathrm{mM}$ $p$-nitrophenyl phosphate, $20 \mathrm{mM} \mathrm{NaF}$, and $20 \mathrm{mM} \beta$ glycerophosphate. To purify the kinases, $\mathrm{Ni}^{2+}$-charged immobilized metal-chelating Sepharose beads (Amersham Biosciences) were added to the cell extracts containing $1.5 \mathrm{mg}$ protein, and the protein-bound beads were used directly in kinase assays. GST-tagged proteins were expressed in Escherichia coli BL21 (DE3) cells transformed with the appropriate cDNA plasmid constructs. Expression was induced with $0.4 \mathrm{mM}$ isopropyl- $\beta$-Dthiogalactopyranoside, and proteins were purified with glutathione-Sepharose beads (Amersham Biosciences).

Indirect Immunofluorescence Microscopy-Indirect immunofluorescence analysis was performed as described

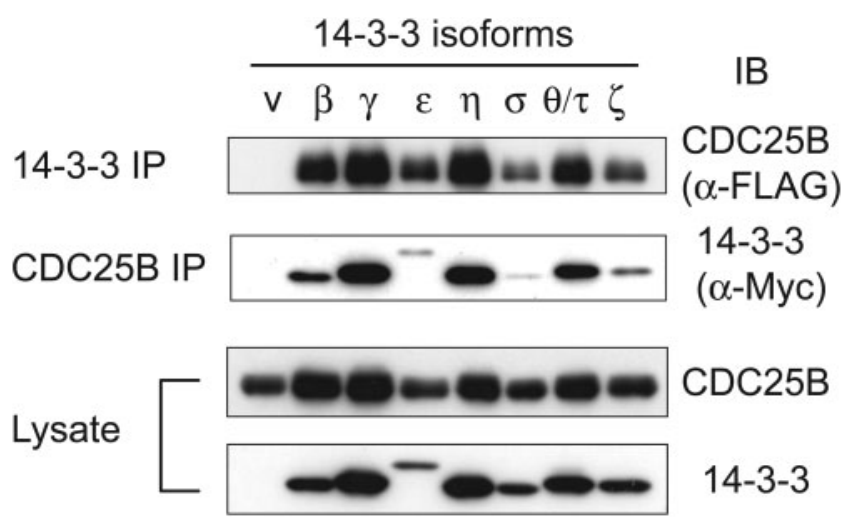

Fig. 1. Binding of 14-3-3 subtypes to CDC25B. HEK293 cells $\left(1.4 \times 10^{6}\right.$ cells per $35-\mathrm{mm}$ plate) were transfected with $3 \mu \mathrm{g}$ of FLAG-tagged CDC25B DNA and 0.6 $\mu \mathrm{g}$ of myc-tagged 14-3-3 DNA. The cells were collected $24 \mathrm{~h}$ after transfection, and cell extracts were prepared. The cell extracts were either subjected to Western blotting to visualize protein expression or further processed for immunoprecipitation with anti-FLAG or anti-myc antibodies to analyze binding. Upper panel: IP with anti-myc (14-3-3), followed by IB with anti-FLAG (CDC25B); second panel: IP with anti-FLAG (CDC25B), followed by IB with anti-myc (14-3-3).

previously (30, 37). Transfected HEK293 cells were fixed with $3.7 \%$ formaldehyde and then permeabilized with $0.5 \%$ Triton X-100. Expressed CDC25B proteins were detected with rabbit polyclonal anti-FLAG antibodies, followed by AlexaFluor 594- or 488-conjugated goat anti-rabbit IgG (Molecular Probes, Eugene, OR, USA), and expressed 143-3 was detected with mouse monoclonal anti-Myc antibodies and AlexaFluor 488-conjugated goat anti-mouse IgG. Nuclei were stained with $4^{\prime}, 6$-diamino-2-phenylindole (Sigma).

\section{RESULTS}

All Seven Isoforms of 14-3-3 Bind to CDC25B-We previously showed that 14-3-3 isoforms $\beta, \varepsilon$, and $\sigma$ bind efficiently to CDC25B, with the $\beta$ and $\varepsilon$ isoforms binding preferentially to the 14-3-3 binding motif at Ser309 (309 site), and the $\sigma$ isoform binding preferentially to the motif at Ser216 (216 site) (30). In the present study, we analyzed the binding properties of four additional isoforms of 14-3-3. The genes for the $\gamma, \eta, \theta$, and $\zeta$ isoforms were amplified by PCR and expressed in HEK293 cells. Each 14-3-3 isoform was co-expressed with CDC25B, isolated, and analyzed as to its interaction with CDC25B. As shown in Fig. 1, all the14-3-3 isoforms were able to interact with CDC25B. CDC25B was detected in immunoprecipitates of all the 14-3-3 isoforms (Fig. 1, upper panel), and all the 14-3-3 isoforms were recovered in the CDC25B co-immunoprecipitates (Fig. 1, second panel). Taking the protein expression levels into account, the $14-3-3 \varepsilon, \sigma$, and $\zeta$ isoforms appeared to bind more weakly to CDC25B than did the other 14-3-3 isoforms (Fig. 1, second and fourth panels).

As we reported previously (30), CDC25B has five 14-3-3 consensus binding motifs (Fig. 2A). Here, we examined the CDC25B binding site preference of each 14-3-3 isoform using co-transfection with plasmids encoding various CDC25B mutants. Five of these mutants contained 


\begin{tabular}{lrlllllll} 
A & \multicolumn{10}{c}{ Consensus } \\
& & R & S & x & pS/T & x & P & \\
81Ser & 78 & R & R & A & $\mathbf{8}$ & E & S & 83 \\
137Ser & 134 & R & F & Q & $\mathbf{8}$ & M & P & 139 \\
216Ser & 213 & R & P & S & $\mathbf{8}$ & A & P & 218 \\
309Ser & 306 & R & S & P & $\mathbf{8}$ & M & P & 311 \\
361Ser & 358 & R & S & K & $\mathbf{8}$ & L & C & 363
\end{tabular}

B

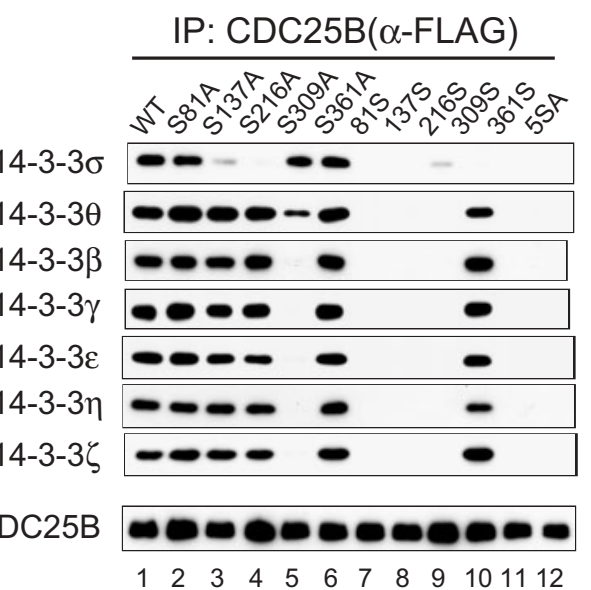

\begin{tabular}{|c|c|c|c|c|c|c|c|c|c|c|c|c|}
\hline \multirow{2}{*}{$\begin{array}{c}14-3-3 \\
\text { subtype }\end{array}$} & \multicolumn{12}{|c|}{ CDC25B mutants } \\
\hline & WT & S81A & S137A & $\mathrm{S} 216 \mathrm{~A}$ & S309A & S361A & $81 \mathrm{~S}$ & 1375 & $216 \mathrm{~S}$ & 3095 & $361 \mathrm{~S}$ & $5 \mathrm{SA}$ \\
\hline$\sigma$ & ++ & ++ & + & - & ++ & ++ & - & - & \pm & - & $\begin{array}{l}- \\
-\end{array}$ & - \\
\hline$\theta$ & ++ & ++ & ++ & ++ & + & ++ & - & - & - & ++ & - & - \\
\hline$\beta$ & ++ & ++ & ++ & ++ & - & ++ & - & - & - & ++ & - & - \\
\hline$\gamma$ & ++ & ++ & ++ & ++ & \pm & ++ & - & - & - & ++ & $\overline{-}$ & - \\
\hline$\varepsilon$ & ++ & ++ & ++ & ++ & \pm & ++ & - & - & - & ++ & - & - \\
\hline$\eta$ & ++ & ++ & ++ & ++ & - & ++ & - & - & - & ++ & - & - \\
\hline$\zeta$ & ++ & ++ & ++ & ++ & \pm & ++ & - & - & - & ++ & - & - \\
\hline
\end{tabular}

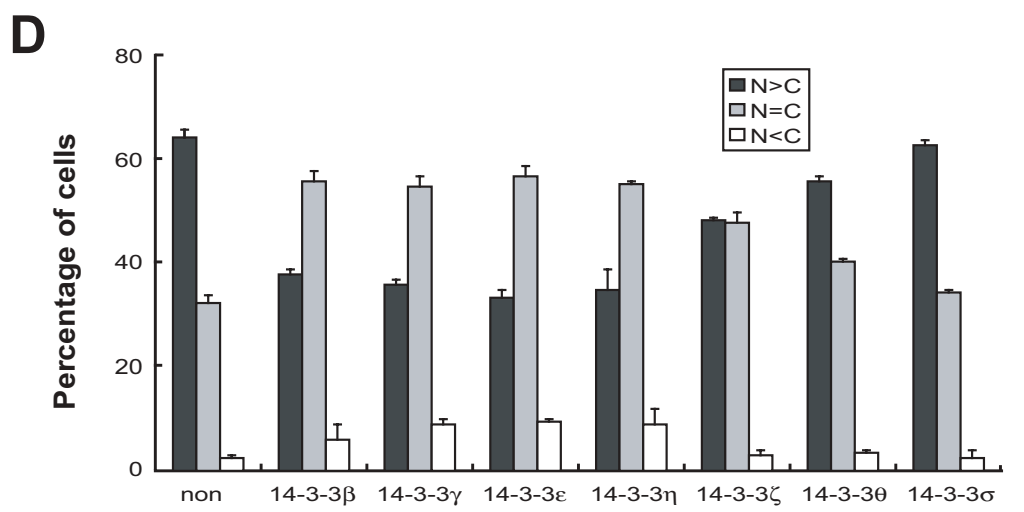

Fig. 2. CDC25B binding site preferences of 14-3-3 subtypes. FLAG-tagged CDC25B mutants with $\mathrm{Ser} \rightarrow$ Ala substitutions at possible 14-3-3 binding sites (30) were co-transfected with each 14-3-3 subtype, as described in Fig. 1. Cell extracts were prepared $24 \mathrm{~h}$ after transfection and then immunoprecipitated with antiFLAG-agarose beads to recover the CDC25B mutant proteins. Recovered CDC25B and co-immunoprecipitated 14-3-3 were detected by Western blotting. A: Possible 14-3-3 consensus binding sites and their amino acid sequences. B: Isoforms of 14-3-3 recovered with anti-FLAG-agarose beads. The bottom panel indicates a typical result of expression of co-transfected CDC25B mutants

C. Summary of the results shown in B. ++, strong signal; +, moderate signal; \pm , discernible after long exposure;-, no signal. D. Subcellular localization of wild-type CDC25B upon co-expression with 14-3-3 subtypes. Cover slips in 35-mm plates were seeded with $2 \times 10^{5}$ HEK293 cells. After 2 days, the cells were transfected with myc-tagged 14-3-3 subtypes in combination with FLAG-tagged wild-type CDC25B. The cells were fixed $24 \mathrm{~h}$ after transfection, and the subcellular localization of CDC25B was determined by staining with anti-FLAG antibodies. More than 200 cells were examined. The data shown are the averages of three independent experiments. Bars = standard deviation.

single-site $\mathrm{Ser} \rightarrow$ Ala substitutions in one 14-3-3 binding motif (e.g., mutant Ser309Ala). In addition, the 309Ser mutant contained $\mathrm{Ser} \rightarrow$ Ala substitutions at all possible binding sites except site 309, and the 5SerAla mutant contained Ser $\rightarrow$ Ala substitutions at all five putative binding sites.

CDC25B immunoprecipitates were recovered from cells co-transfected with the 14-3-3 and mutant CDC25 genes, and 14-3-3 was detected on Western blots. All 14-3-3 isoforms except 14-3-3 $\sigma$ were found to bind preferentially to site 309 (Fig. 2B), as shown previously (30). Although substantial binding of $14-3-3 \theta$ to the Ser309Ala mutant did occur, comparison with the other single-site mutations revealed that 14-3-30 bound preferentially to site 309 . These results are summarized in Fig. 2C.

We examined the effect of co-transfection with 14-3-3 isoforms on the subcellular localization of CDC25B. Previously, we found that the binding of 14-3-3 at site 309 caused CDC25B to move from the nucleus to the cytoplasm (30). In the present study, we found that 14-3-3 isoforms that preferentially bound at site 309 caused CDC25B to be exported from the nucleus to the cytoplasm, and that isoforms that bound weakly or nonpreferentially to site 309 did not have such an effect
(Fig. 2D). The 14-3-30 isoform, which binds preferentially to site 309, exhibited a mobilizing effect on the 309Ser mutant (data not shown), consistent with the results of the binding analysis above.

Mutations Near Ser309 of CDC25B Interfere with 14-3-3 binding-The effects of co-transfection with 14-3-3 on the cytoplasmic distribution of CDC25B indicated that 14-3-3 binding at site 309 may have masked the nuclear localization signal (NLS) sequence of CDC25B, which begins about ten residues downstream of the binding site (Fig. 3A). We therefore conducted experiments to determine the effect of an increased distance between Ser309 and the NLS sequence. Lys320 is the first residue of the bipartite NLS sequence in CDC25B. To enable the in-frame insertion of additional amino acids, we initially used mutagenesis to introduce a BamHI site between residues 319 and 320 . The BamHI recognition sequence resulted in the insertion of Gly-Ser (GS) dipeptide immediately before Lys320 (Fig. 3A). We found, however, that this GS insertion abolished 14-3-3 binding to CDC25B, despite the fact that phosphorylation at site 309 was detected with phospho-Ser309 antibodies (Fig. 3B).

These results were intriguing because there have been no previous reports that amino acid sequences C-terminal 
A

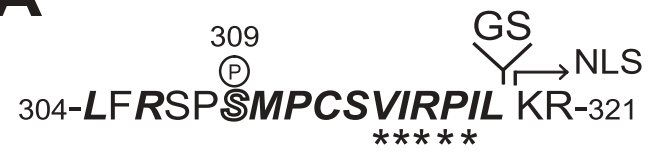

B

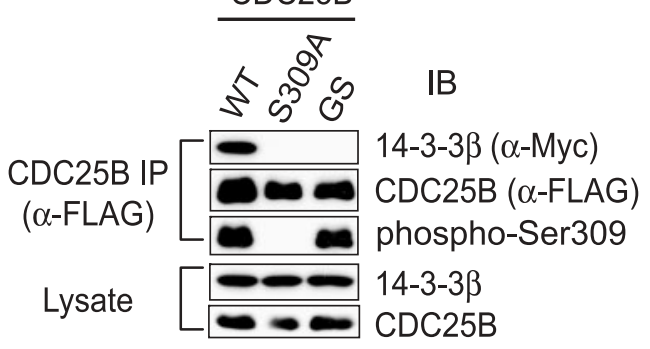

C

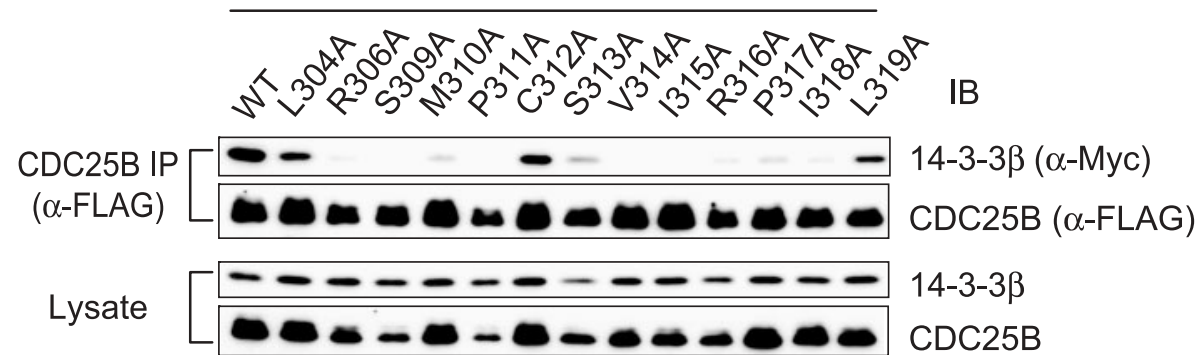

D

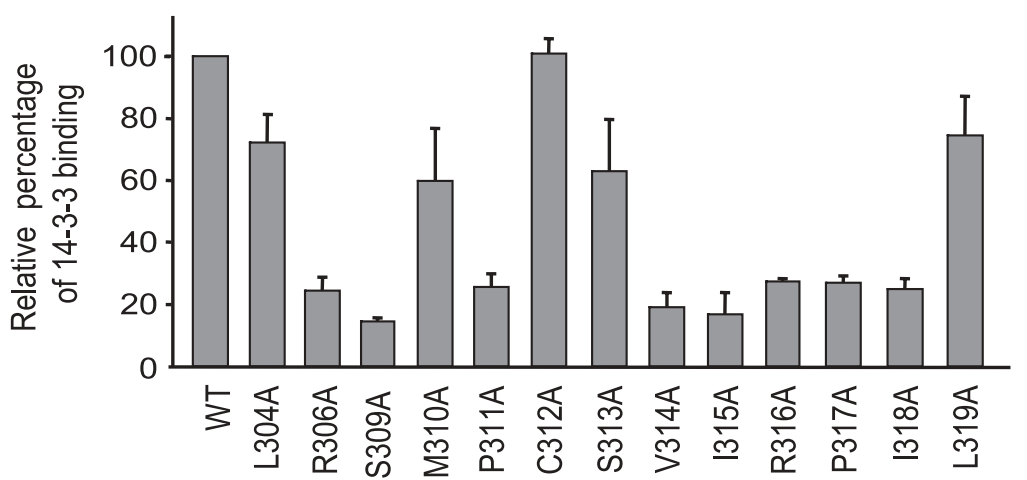

Fig. 3. Binding of 14-3-3 $\beta$ to CDC25B mutants with substitutions near Ser309. CDC25B mutants containing point substitutions near Ser309 were

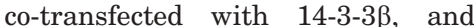
CDC25B mutant proteins were isolated with anti-FLAG-agarose beads as described in Fig. 2 CDC25B-bound 14-3-3 $\beta$ was identified with an anti-myc antibody. A. Amino acid sequence from Leu304 to Arg321. Ser309 is denoted by an outlined letter S. A BamHI site (GGATCC), which encodes GlySer, was inserted between Leu319 and Lys320. Lys 320 is the N-terminal end of the NLS sequence in CDC25B, which is indicated by an arrow. Bold italic letters indicate amino acids changed to Ala to analyze 14-3-3 binding, the results of which are depicted in (C) and (D). Asterisks indicate the hydrophobic amino acid stretch (see text). The GS insertion mutant (+ GS in B) and a series of point mutants (C) of CDC25B were analyzed as to their binding to 14-3$3 \beta$ and the phosphorylation of Ser309, which was determined with anti-phospho-Ser309 antibodies. The efficiency of 14-3-3 $\beta$ binding to each CDC25B mutant relative to wild-type CDC25B was calculated from the data presented in (C) and is depicted in (D). The data shown are the averages of three independent experiments. $\quad$ Bars $=$ standard deviation. to the 14-3-3 binding site affect the interaction between 14-3-3 and its target proteins. We further examined this phenomenon by introducing single alanine point mutations at residues 304 to 319 of CDC25B, and then co-transfecting the resulting mutants and 14-3-3 $\beta$ (indicated by bold italic letters in Fig. 3A). Surprisingly, all of the tested point mutations except Cys312Ala and Leu319Ala caused a diminished interaction with 14-3-3 (Fig. 3C). The results clearly demonstrate that the hydrophobic amino acid region from Val315 to Ile318 seems to be important for 14-3-3 binding (Fig. 3D and depicted by asterisks in Fig. 3A). Pro311 of CDC25B, which is part of the 14-3-3 binding consensus sequence, also appeared to be important for 14-3-3 binding. Thus, alterations in the amino acids near the core consensus sequence of site 309 of CDC25B negatively affected its ability to bind to 14-3-3.

Ser309 Phosphorylation by Several Candidate Kinases-To study the in vivo phosphorylation of Ser309 in these mutants, we transfected some of the mutant $C D C 25 B$ genes, and the mutant proteins were recovered and assayed for phosphorylation at Ser309. As shown in Fig. 4A, phosphorylation of Ser309 occurred in all of the mutants expressed in HEK293 cells, except for the Pro317Ala mutant expressed in Cos-7 cells.

Several kinases that phosphorylate Ser309 of CDC25B1 (or Ser323 of CDC25B3) have been reported. We attempted to determine which kinases could phosphorylate the CDC25B mutants. We expressed candidate kinases 


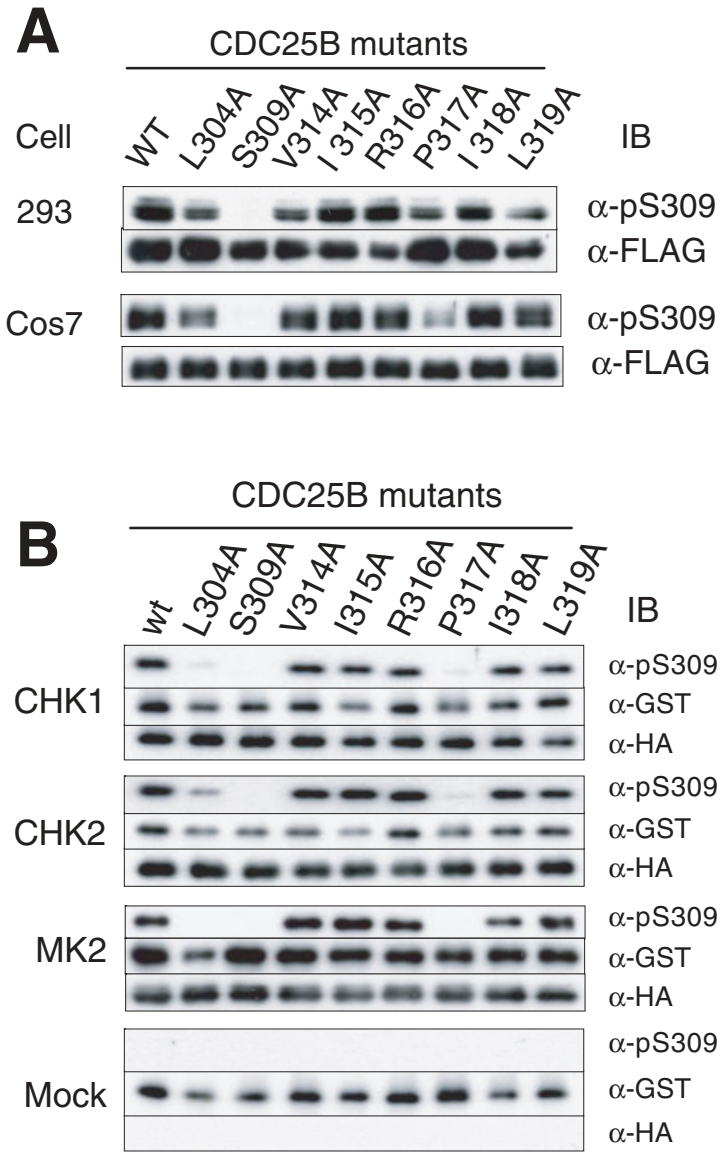

Fig. 4. Phosphorylation of Ser309 in vivo and in vitro by representative kinases. HEK293 cells $\left(1.4 \times 10^{6}\right.$ cells per $35-\mathrm{mm}$ plate $)$ or Cos-7 cells $\left(2 \times 10^{5}\right.$ cells per $35-\mathrm{mm}$ plate $)$ were transfected with plasmids carrying FLAG-tagged CDC25B mutants, and the expressed proteins were immunoprecipitated with monoclonal anti-FLAG beads. The precipitated proteins were immunoblotted with anti-FLAG or anti-phospho-Ser309 antibodies (A). His- and HA-tagged kinases were expressed in Cos-7 cells and recovered as described. The recovered kinases were incubated with GST-fused CDC25B peptides (wild-type or mutant), and phosphorylation at Ser309 was determined by immunoblotting (B).

CHK1 (18), CHK2 (19) and MK2 (26) in Cos-7 cells and then recovered the kinases by immunoprecipitation. For the preparation of $\mathrm{MK} 2$, an upstream kinase, mitogenactivated protein kinase kinase kinase 6 (MKK6) and MK2 were co-transfected, and the expressed MK2 was immunoprecipitated. An in vitro phosphorylation assay indicated that the three kinases could phosphorylate GST-tagged CDC25B mutant peptides (Fig. 4B), the results being similar to those obtained in vivo (Fig. 4A). The kinases phosphorylated all of the mutant peptides with similar efficiency, with the exception of the Pro317Ala mutant, consistent with the results shown in Fig. 4A. These results demonstrate that Ser309 of CDC25B can be phosphorylated by several kinases.

Binding of Endogenous 14-3-3 to CDC25B Mutants and Their Subcellular Localization-We examined the binding of endogenous 14-3-3 to mutant CDC25B proteins. After transfection to HEK293 cells, CDC25B proteins were
A

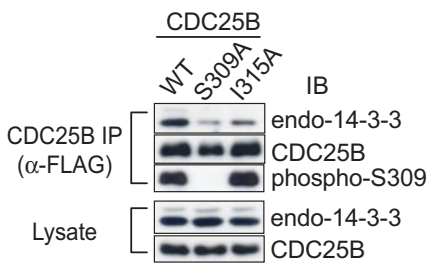

B

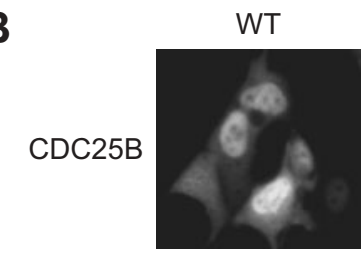

Nuclear

Iolalization (\%): $\quad 60.1 \pm 2.1$

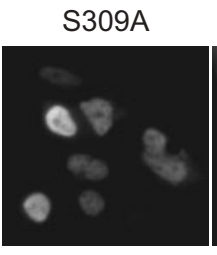

$1315 \mathrm{~A}$

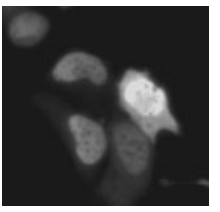

$94.7 \pm 2.1$

$72.3 \pm 1.0$

Fig. 5. Binding of endogenous 14-3-3 to CDC25B mutants and subcellular localization. HEK293 cells were transfected with plasmids carrying wild type CDC25B or Ala309 or Ala315 mutants, and the expressed proteins were recovered with antiFLAG beads. The precipitates were immunoblotted with an antipan 14-3-3 antibody (upper panel), anti-FLAG antibody (second panel), or anti-phospho-Ser309 antibody (third panel). The expression of endogenous 14-3-3 and transfected CDC25B is indicated in the two bottom panels (A). HEK293 cells were transfected with CDC25B plasmids as in (A), and the subcellular localization of the expressed CDC25B proteins was determined as in Fig. 2D. The numbers represent the percentages of cells expressing the CDC25B protein found exclusively in the nucleus. Three independent experiments were performed for each value, and more than 200 CDC25B-expressing cells were counted in each experiment (B).

recovered, and the bound 14-3-3 protein was quantitated using a pan-14-3-3 antibody. Figure 5A shows that the efficiency of endogenous 14-3-3 binding to mutant CDC25B was similar to that in the co-expression experiments. Endogenous 14-3-3 bound to wild-type CDC25B most efficiently and to the 309Ala mutant least efficiently. A lesser amount of endogenous 14-3-3 was detected with the 315Ala mutant, which exactly matched the results in Fig. 3C and D. The phosphorylation of S309 in the I315A mutant could also be reproduced (compare Figs. 5A and 4A). We examined the subcellular localization of these mutant CDC25B proteins expressed in cells (Fig. 5B). The number of cells with CDC25B specifically localized in the nucleus was higher for mutant CDC25B than for the wild-type protein. About $72 \%$ of the cells exhibited specific nuclear localization of CDC25B 315Ala mutants, which was higher than the frequency of nuclear localization of wild-type CDC25B ( 60\%). Collectively, amino acid sequences surrounding the 14-3-3 binding core consensus site have a strong influence on 14-3-3 binding but subcellular localization of CDC25B can not be simply explained by 14-3-3 binding.

Finally, the results of this study are summarized in Table 3.

\section{DISCUSSION}

Seven isoforms of 14-3-3 have been identified in mammalian cells. We previously reported that three isoforms, 14-3$3 \beta, \varepsilon$, and $\sigma$, are able to bind to $\mathrm{CDC} 25 \mathrm{~B}$, and that the 
Table 3. Summary of 14-3-3 $\beta$ binding and Ser309 phosphorylation of each CDC25B mutant.

\begin{tabular}{|c|c|c|c|c|}
\hline \multirow[t]{2}{*}{ Name } & \multirow[t]{2}{*}{ Sequence } & \multirow{2}{*}{$\begin{array}{c}14-3-3 \beta \\
\text { Binding }(\%)\end{array}$} & \multicolumn{2}{|c|}{ Ser309 phosphorylation } \\
\hline & & & in vivo & in vitro \\
\hline$\overline{\mathrm{WT}}$ & ${ }^{304}{ }^{2 F R S P S M P C S V I R P I L K R ~}{ }^{321}$ & 100 & + & + \\
\hline GS & LFRSPSMPCSVIRPILGSKR & 17 & + & NT \\
\hline L304A & $\mathrm{A}-\ldots-\ldots-\ldots-\ldots R$ & 70 & + & \pm \\
\hline R306A & - -A- - - - - - - - -KR & 23 & NT & NT \\
\hline S309A & 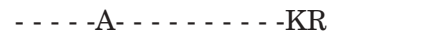 & 18 & - & - \\
\hline M310A & - - - - - - - - - - -KR & 60 & NT & NT \\
\hline P311A & - - - - - - - - - -KR & 25 & NT & NT \\
\hline C312A & - - - - - - - - - - KR & 103 & NT & NT \\
\hline S313A & - - - - - - - - - - -KR & 63 & NT & NT \\
\hline V314A & - - - - - - - - - -KR & 21 & + & + \\
\hline I $315 \mathrm{~A}$ & - - - - - - - - - -KR & 17 & + & + \\
\hline R316A & - - - - - - - - - -KR & 27 & + & + \\
\hline P317A & - - - - - - - -A- -KR & 27 & + & \pm \\
\hline I318A & 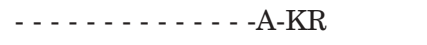 & 25 & + & + \\
\hline L319A & 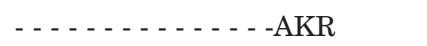 & 72 & + & + \\
\hline
\end{tabular}

binding site for 14-3-3 $\beta$ and $\varepsilon$ is different from that for 14-3$3 \sigma$. Moreover, after binding to $14-3-3 \beta$ or $\varepsilon$ but not $\sigma$, CDC25B is exported to the cytoplasm. The recently published X-ray structure results revealed the following specific functional and structural features of the 14-3-3 $\sigma$ isoform. First, it usually forms a homodimer. Second, 14$3-3 \sigma$ possesses a ligand-discriminating, special amino acid patch on the second ligand-binding surface (38). These findings may explain the difference in the binding properties of 14-3-3 $\beta$ and $\sigma$ as to CDC25B.

Here, we evaluated whether four other 14-3-3 subtypes $(\gamma, \eta, \theta / \tau$, and $\zeta$ ) also bind to CDC25B and exhibit behavior similar to that of $14-3-3 \beta$. The $14-3-3 \theta / \tau$ isoform exhibited slightly different properties from those of the other three isoforms in that it bound to other 14-3-3 motifs in CDC25B in addition to site 309. The 14-3-3ל isoform bound to CDC25B more weakly at Ser309 than did the other isoforms, except 14-3-3 $\sigma$. These subtle differences appeared to affect the ability of these isoforms to cause the transfer of CDC25B from the nucleus to the cytoplasm. Our results show that six of the seven 14-3-3 subtypes in mammalian cells behave similarly in terms of CDC25B binding. The specific function of the $14-3-3 \sigma$ isoform is unknown.

The typical consensus 14-3-3 binding sequence consists of Arg-X-X-Ser/Thr-X-P (mode-I) or Arg-X-X-X-Ser/Thr-X$\mathrm{P}$ (mode-II), where the serine or threonine must be phosphorylated $(39,40)$. In addition to these binding motifs, 14-3-3 also binds to the recently identified mode-III motif, which consists of (p)S/T-X $\mathrm{X}_{1-2}-\mathrm{COOH}$ at the C-terminus of several proteins (41). Although the results of oriented peptide analysis suggest that the Arg-X-X(p)Ser/Thr-X-P mode-I motif is sufficient for 14-3-3 binding, a more complicated situation appears to exist in vivo. For example, we previously found that CDC25B contains five 14-3-3 binding motifs, but only three of these are functional. The principal 14-3-3 binding site is at Ser309, and the Ser216 site in combination with the Ser137 or Ser309 site may also be important, especially for binding to $14-3-3 \sigma$.

One possible reason for the apparent non-functionality of certain 14-3-3 binding motifs in vivo may be the lack of phosphorylation of target Ser/Thr. The results described in this report suggest another somewhat unexpected explanation. We found that alterations in amino acids C-terminal to the binding motif at site 309 severely impaired 14-3-3 binding, indicating that 14-3-3 binding depends not only on the consensus sequence but also on its context. Thus, the binding of 14-3-3 may require not only the presence of the binding motif in its appropriately phosphorylated form but also the presence of a suitable sequence C-terminal to the binding site. Substitution mutations of amino acid residues often disrupt the local structure of proteins. Therefore, some mutants examined in this study may harbor a structural change that abolishes 14-3-3 binding. However, Ser309 in each CDC25B mutant, except Pro317Ala, was efficiently phosphorylated at the same level as that of the wild-type protein in transfected cells. These results imply (but do not prove unequivocally) that a gross structural change should not be introduced by the mutations. Studies using an oriented peptide library with a random amino acid sequence placed C-terminal to the consensus site will be important for confirmation of our hypothesis.

The specific kinases that phosphorylate Ser309 of CDC25B have yet to be identified. We examined kinases that have been reported to phosphorylate this residue, but they exhibited essentially the same specificity toward mutated substrates in vitro. All of the tested kinases required a proline at residue 317 and an arginine at residue 306, although Pro317 was not essential for Ser309 phosphorylation in vivo. The kinase responsible for regulating CDC25B localization must exhibit an appropriate subcellular localization. In the normal cell cycle, CDC25B is located in the nucleus, and Ser309 is phosphorylated to some degree even in the absence of cellular injury (23). In view of this, kinases that phosphorylate Ser309 would also be expected to be located in the nucleus. Of these kinases, we propose that CHK1 and MK2 may be suitable for the phosphorylation of Ser309 of CDC25B1, even in the absence of specific cellular damage, since they are activated at low levels during DNA replication and environmental stress, respectively (Ref. 26 and our unpublished data). It is a matter for further investigation 
as to whether these kinases phosphorylate this site more heavily when cells are injured.

We thank S. Elledge for the generous gift of the plasmids. This work was supported by Grants-in-Aid for Scientific Research from and by the Third-Term Comprehensive 10-Year Strategy for Cancer Control of the Ministry of Health, Labour, and Welfare of Japan. This work was also supported by the Collaborative Research Program of the Cancer Institute of Kanazawa University and by the Kanazawa University 21st Century COE Program of the Ministry of Education, Culture, Sports, Science, and Technology of Japan. S. U. was also supported by a fellowship from the Kanazawa University 21st Century COE Program and a Research-Resident Fellowship from the Human Science Foundation of Japan.

\section{REFERENCES}

1. Galaktionov, K. and Beach, D. (1991) Specific activation of cdc25 tyrosine phosphatases by B-type cyclins: evidence for multiple roles of mitotic cyclins. Cell 67, 1181-1194

2. Sadhu, K., Reed, S.I., Richardson, H., and Russell, P. (1990) Human homolog of fission yeast cdc25 mitotic inducer is predominantly expressed in G2. Proc. Natl. Acad. Sci. USA 87, 5139-5143

3. Strausfeld, U., Labbe, J.C., Fesquet, D., Cavadore, J.C., Picard, A., Sadhu, K., Russell, P., and Doree, M. (1991) Dephosphorylation and activation of a p34cdc2/cyclin B complex in vitro by human CDC25 protein. Nature 351, 242-245

4. Jinno, S., Suto, K., Nagata, A., Igarashi, M., Kanaoka, Y., Nojima, H., and Okayama, H. (1994) Cdc25A is a novel phosphatase functioning early in the cell cycle. EMBO J. 13 , 1549-1556

5. Hoffmann, I., Draetta, G., and Karsenti, E. (1994) Activation of the phosphatase activity of human cdc25A by a cdk2-cyclin $\mathrm{E}$ dependent phosphorylation at the G1/S transition. EMBO J. 13, 4302-4310

6. Kumagai, A. and Dunphy, W.G. (1992) Regulation of the cdc25 protein during the cell cycle in Xenopus extracts. Cell 70, 139-151

7. Hoffmann, I., Clarke, P.R., Marcote, M.J., Karsenti, E., and Draetta, G. (1993) Phosphorylation and activation of human cdc25-C by cdc2-cyclin B and its involvement in the selfamplification of MPF at mitosis. EMBO J. 12, 53-63

8. Nishijima, H., Nishitani, H., Seki, T., and Nishimoto, T. (1997) A dual-specificity phosphatase Cdc25B is an unstable protein and triggers p34(cdc2)/cyclin B activation in hamster BHK21 cells arrested with hydroxyurea. J. Cell Biol. 138, 1105-1116

9. Lammer, C., Wagerer, S., Saffrich, R., Mertens, D., Ansorge, W., and Hoffmann, I. (1998) The cdc25B phosphatase is essential for the G2/M phase transition in human cells. J. Cell Sci. 111, 2445-2453

10. Donzelli, M., Squatrito, M., Ganoth, D., Hershko, A., Pagano, M., and Draetta, G.F. (2002) Dual mode of degradation of Cdc25 A phosphatase. EMBO J. 21, 4875-4884

11. Mailand, N., Podtelejnikov, A.V., Groth, A., Mann, M., Bartek, J., and Lukas, J. (2002) Regulation of G(2)/M events by Cdc25A through phosphorylation-dependent modulation of its stability. EMBO J. 21, 5911-5920

12. Chen, M.S., Hurov, J., White, L.S., Woodford-Thomas, T., and Piwnica-Worms, H. (2001) Absence of apparent phenotype in mice lacking Cdc25C protein phosphatase. Mol. Cell. Biol. 21, 3853-3861

13. Lincoln, A.J., Wickramasinghe, D., Stein, P., Schultz, R.M., Palko, M.E., De Miguel, M.P., Tessarollo, L., and Donovan, P.J. (2002) Cdc25b phosphatase is required for resumption of meiosis during oocyte maturation. Nat. Genet. 30, 446-449
14. Ferguson, A.M., White, L.S., Donovan, P.J., and Piwnica-Worms, H. (2005) Normal cell cycle and checkpoint responses in mice and cells lacking Cdc25B and Cdc25C protein phosphatases. Mol. Cell. Biol. 25, 2853-2860

15. Bulavin, D.V., Amundson, S.A., and Fornace, A.J. (2002) p38 and Chk1 kinases: different conductors for the $\mathrm{G}(2) / \mathrm{M}$ checkpoint symphony. Curr. Opin. Genet. Dev. 12, 92-97

16. Donzelli, M. and Draetta, G.F. (2003) Regulating mammalian checkpoints through Cdc25 inactivation EMBO Rep. 4, 671-677

17. Bartek, J., Lukas, C., and Lukas, J. (2004) Checking on DNA damage in S phase. Nat. Rev. Mol. Cell. Biol. 5, 792-804

18. Sanchez, Y., Wong, C., Thoma, R.S., Richman, R., Wu, Z., Piwnica-Worms, H., and Elledge, S.J. (1997) Conservation of the Chk1 checkpoint pathway in mammals: linkage of DNA damage to Cdk regulation through Cdc25. Science 277, 1497-1501

19. Matsuoka, S., Huang, M., and Elledge, S.J. (1998) Linkage of ATM to cell cycle regulation by the Chk2 protein kinase. Science 282, 1893-1897

20. O’Neill, T., Giarratani, L., Chen, P., Iyer, L., Lee, C.H., Bobiak, M., Kanai, F., Zhou, B.B., Chung, J.H., and Rathbun, G.A. (2002) Determination of substrate motifs for human Chk1 and hCds1/Chk2 by the oriented peptide library approach. J. Biol. Chem. 277, 16102-16115

21. Miyata, H., Doki, Y., Yamamoto, H., Kishi, K., Takemoto, H., Fujiwara, Y., Yasuda, T., Yano, M., Inoue, M., Shiozaki, H., Weinstein, I.B., and Monden, M. (2001) Overexpression of CDC25B overrides radiation-induced G2-M arrest and results in increased apoptosis in esophageal cancer cells. Cancer Res. 61, 3188-3193

22. Forrest, A. and Gabrielli, B. (2001) Cdc25B activity is regulated by 14-3-3. Oncogene 20, 4393-4401

23. Bulavin, D.V., Higashimoto, Y., Popoff, I.J., Gaarde, W.A., Basrur, V., Potapova, O., Appella, E., and Fornace, A.J., Jr. (2001) Initiation of a G2/M checkpoint after ultraviolet radiation requires p38 kinase. Nature 411, 102-107

24. Peng, C.Y., Graves, P.R., Thoma, R.S., Wu, Z., Shaw, A.S., and Piwnica-Worms, H. (1997) Mitotic and G2 checkpoint control: regulation of 14-3-3 protein binding by phosphorylation of Cdc25C on serine-216. Science 277, 1501-1505

25. Giles, N., Forrest, A., and Gabrielli, B. (2003) 14-3-3 acts as an intramolecular bridge to regulate cdc25B localization and activity. J. Biol. Chem. 278, 28580-28587

26. Manke, I.A., Nguyen, A., Lim, D., Stewart, M.Q., Elia, A.E., and Yaffe, M.B. (2005) MAPKAP kinase-2 is a cell cycle checkpoint kinase that regulates the G2/M transition and $\mathrm{S}$ phase progression in response to UV irradiation. Mol. Cell 17, $37-48$

27. Dalal, S.N., Schweitzer, C.M., Gan, J., and DeCaprio, J.A. (1999) Cytoplasmic localization of human cdc25C during interphase requires an intact 14-3-3 binding site. Mol. Cell. Biol. 19 $4465-4479$

28. Davezac, N., Baldin, V., Gabrielli, B., Forrest, A., Theis-Febvre, N., Yashida, M., and Ducommun, B. (2000) Regulation of CDC25B phosphatases subcellular localization. Oncogene 19, 2179-2185

29. Graves, P.R., Lovly, C.M., Uy, G.L., and Piwnica-Worms, H. (2001) Localization of human Cdc25C is regulated both by nuclear export and 14-3-3 protein binding. Oncogene 20, $1839-1851$

30. Uchida, S., Kuma, A., Ohtsubo, M., Shimura, M., Hirata, M., Nakagama, H., Matsunaga, T., Ishizaka, Y., and Yamashita, K. (2004) Binding of 14-3-3beta but not 14-3-3sigma controls the cytoplasmic localization of CDC25B: binding site preferences of 14-3-3 subtypes and the subcellular localization of CDC25B. J. Cell Sci. 117, 3011-3020

31. Furnari, B., Blasina, A., Boddy, M.N., McGowan, C.H., and Russell, P. (1999) Cdc25 inhibited in vivo and in vitro by checkpoint kinases Cds1 and Chk1. Mol. Biol. Cell 10, 833-845 
32. Chen, M.S., Ryan, C.E., and Piwnica-Worms, H. (2003) Chk1 kinase negatively regulates mitotic function of Cdc25A phosphatase through 14-3-3 binding. Mol. Cell. Biol. 23, $7488-7497$

33. Uto, K., Inoue, D., Shimuta, K., Nakajo, N., and Sagata, N. (2004) Chk1, but not Chk2, inhibits Cdc25 phosphatases by a novel common mechanism. EMBO J. 23, 3386-3396

34. Guan, K.L. and Dixon, J.E. (1991) Eukaryotic proteins expressed in Escherichia coli: an improved thrombin cleavage and purification procedure of fusion proteins with glutathione S-transferase. Anal. Biochem. 192, 262-267

35. Wang, X., Arooz, T., Siu, W.Y., Chiu, C.H., Lau, A., Yamashita, K., and Poon, R.Y. (2001) MDM2 and MDMX can interact differently with $\mathrm{ARF}$ and members of the p53 family. FEBS Lett. 490, 202-208

36. Bradford, M.M. (1976) A rapid and sensitive method for the quantitation of microgram quantities of protein utilizing the principle of protein-dye binding. Anal. Biochem. 72, 248-254
37. Uchida, S., Ohtsubo, M., Shimura, M., Hirata, M., Nakagama, H., Matsunaga, T., Yoshida, M., Ishizaka, Y., and Yamashita, K. (2004) Nuclear export signal in CDC25B. Biochem. Biophys. Res. Commun. 316, 226-232

38. Wilker, E.W., Grant, R.A., Artim, S.C., and Yaffe, M.B. (2005) A structural basis for 14-3-3sigma functional specificity. J. Biol. Chem. 280, 18891-18898

39. Muslin, A.J., Tanner, J.W., Allen, P.M., and Shaw, A.S. (1996) Interaction of 14-3-3 with signaling proteins is mediated by the recognition of phosphoserine. Cell 84, 889-897

40. Yaffe, M.B., Rittinger, K., Volinia, S., Caron, P.R., Aitken, A., Leffers, H., Gamblin, S.J., Smerdon, S.J., and Cantley, L.C. (1997) The structural basis for 14-3-3:phosphopeptide binding specificity. Cell 91, 961-971

41. Ganguly, S., Weller, J.L., Ho, A., Chemineau, P., Malpaux, B., and Klein, D.C. (2005) Melatonin synthesis: 14-33 -dependent activation and inhibition of arylalkylamine $\mathrm{N}$-acetyltransferase mediated by phosphoserine-205. Proc. Natl. Acad. Sci. USA 102, 1222-1227 